\title{
Force Adaptation in Robot Transmissions
}

\author{
Konstantin IVANOV ${ }^{*}$, Marco Ceccarelli ${ }^{2}$ and Baurjan Tultaev ${ }^{3}$ \\ ${ }^{1}$ Almaty University of Power Engineering and Telecommunication, Baytursinov st. 126, Almaty 050013, Kazakhstan \\ ${ }^{2}$ University of Kassino and South Latium, Italy \\ ${ }^{3}$ Djoldasbekov Institute of Mechanics and Machine Science MON RK, Kurmangazy st.19, Almaty 050000, Kazakhstan
}

Received: July 27, 2017; Accepted: August 07, 2017; Published: August 20, 2017

*Corresponding author:Konstantin IVANOV, Professor, Almaty University of Power Engineering and Telecommunication, Baytursinov st. 126, Almaty 050013, Kazakhstan, Tel: +8-727-248-3052, E-mail: ivanovgreek@mail.ru

\begin{abstract}
In this work the theoretical bases of the force adaptation in robot transmissions are stated. The force adaptation in robot transmissions considers creation, research and designing of robots with adaptive electric drives of modules. The adaptive drive contains the electric motor and the adaptive gear mechanism possessing property independently to change output speed of movement depending on loading. This property of adaptation is named as self-regulation. Self-regulation is carried out only at the expense of mechanics and does not demand a control. The adaptive drive demands smaller power, overcomes emergency overloads, is structurally simple and has small dimensions and weight. These advantages are especially important for intermediate modules of the manipulator. Use of adaptive drives provides high power efficiency of the robot and decrease of the sizes.
\end{abstract}

Keywords: Adaptive robot transmissions; Electric drive; Force adaptation;

\section{Introduction}

The drive of the module of the manipulator is performing the starting-up, the steady-state motion and the braking of a moving part of the module at execution of each operation. Speed of motion varies from null to a maximum and back. The greatest loading in the form of an inertia force takes place in the starting-up of operation. The adequate drive should have the variable transfer ratio in order to overcome a start inertia resistance. However application of the adjustable gear box in the manipulator drive is impossible as the drive connecting gear should have small sizes and weight. Therefore the non controllable drive with the constant transfer ratio is used. The engine of the non controllable drive must overcome the short maximum inertial resistance that reduces power efficiency of the drive because in the rest time a loading will be significantly less. Such drive creates dynamic stresses of motion and vibration. At the present time the variable-frequency electric drives of alternating current are finding the more and more wide application in a robotics. They are expelling the uncontrolled electric drives in many areas and also the drives realizing insufficiently economic control modes of speed [1].

The transfer ratio is changed by means of a control system affecting on electric current parameters. In the frequency-operated electric drive the torque is transferring from the engine to loading through the reducer having the constant transfer ratio. But in a robotics it is not possible to use effectively the electric motor and the reducer with the constant transfer ratio as the electric motor cannot be optimized for all operating modes. The electric motor and the reducer must be selected under the maximum torque moment of loading, and in the basic operating modes the drive will essentially underused on power. Therefore it is rational to combine the frequency-controlled engine with a variator.
At frictional disk variators the torque from the input link to the output link is transferring by a frictional force on surfaces of adjoining bodies, and the special control system is necessary for the transfer ratio change. The disk planetary variator is having the restricted application owing to a number of defects. Their overload capacity does not exceed 2-3 and efficiency sharply decreases with loading growth. The majority of existing variators (for example, a disk variator «Disco» of company "Lenze") have a small control range of the transfer ratio and do not admit its change in the disconnect state. Therefore application of such variators is restricted to drives with a small control range of speed and torque.

Gulia's adaptive disk planetary variator allows creating the new class of the electric drives possessing a "soft" external speed-torque characteristic with very high starting torque, overload capacity and possibility of automatic control of the transfer ratio at any changes of loading [1]. However the variator design is very complex and inapplicable for robots.

The toroidal friction variators are no effective for robot transmissions also [2]. $[3,4]$.

The most effective for robots the Ivanov's gear adaptive variators are

The adaptive variator is mechanically simple, has small dimensions and weight and it is working without a control system. These advantages are especially important for intermediate modules of the manipulator. Use of adaptive gear variators provides a high power efficiency of the robot and essential decrease of sizes and weight.

In the electric drive with the adaptive gear variator having a high control range, it is possible to use a usual asynchronous motor. Such drive works without a control system.

The first trying to prove advantage and expediency of application of adaptive drives in a robotics has been made in the work [3]. The researches devoted to creation of adaptive drives of modules of manipulators have been executed. They are allowing a choosing of engine on average (but not on maximal) power of resistance. But this trying was not grounded enough. The adaptive drive has possibility of transition in a "stop" regime with a stop of tool when the engine prolongs to operate [4].

It allows to co-ordinate work of modules at overloading. The "stop" regime can be used also for limiting of force (for example, in grippers).

However power advantages of adaptive drives have not been proved by the kinematic and force analysis.

It is expedient to execute comparison of kinematics of the adaptive drive and the usual drive with "rigid" kinematics for an estimation of advantages of the adaptive drive and also to consider additional 
possibilities of adaptive drives for their use in robot transmissions.

In the present work the adaptive drives of modules of the manipulator are considered. The work and interacting of the adaptive drives is investigated and the basic regularities of interconnection of their parameters are resulted.

\section{Kinematics of a Moving Part of the Module} drive.

The kinematics of the module of the manipulator is defined by its

Now the drive with the constant transfer ratio which defines "rigid" constraint between the engine and a moving part of the module translation or a rotary motion (the drive 1 ) is used. The adaptive drive is alternative to the drive with "rigid" constraint. The adaptive drive has two degrees of freedom and provides motion with the speed depending on loading (the drive 2) $[3,4]$. We will be to consider the kinematics parameters of both used modules of manipulator: module of translation motion and module of rotation motion on one figure. On figure 1 the schedules of linear parameters for module of translation motion and the schedules of angular parameters for module of rotation are presented. On fig. 1 the linear or angular parameters of displacement $(s, \varphi)$, speed $(v, \omega)$ and acceleration $(a, \varepsilon)$ to functions from a time $t$ are presented.

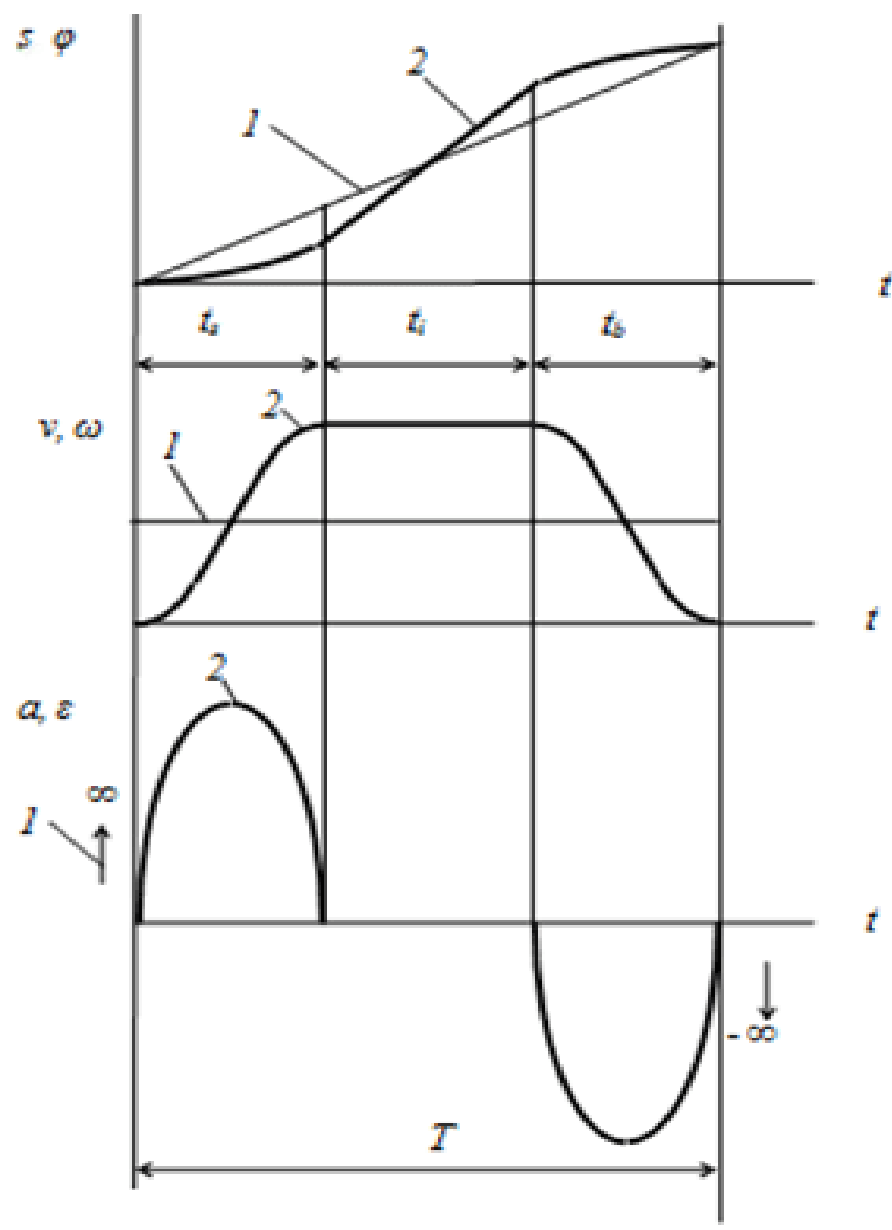

- the drive 1 with "rigid" kinematic constraint - the adaptive drive 2

Figure 1: Kinematics of a moving part of the module of the manipulator

Each carried out operation contains three phases of motion: the start or speeding-up ( $t_{s}$ - start time), the installed motion ( $t_{i}$ - time of installed motion when speed is constant) and the braking ( $t_{b}$ - time of braking). A time of performance of operation $T=t_{s}+t_{i}+t_{b}$

The kinematic schedules for the "rigid" drive 1 are shown by thin lines and for the adaptive drive 2 are shown by contour lines.

Let us to compare the schedules of drive 1 and of drive 2. The comparison of schedules shows the principal advantage of an adaptive drive 2 - possibility to use a drive with considerably smaller power in comparison with the incontrollable drive having a fixed transfer ratio. Usually in the theory of mechanisms the comparison of various laws of motion is used for cyclic cam mechanisms. Also it is possible to consider the manipulator as the cyclic mechanism. The drive 1 with a fixed transfer ratio has accelerations in the beginning and in the end of the removal phase. These accelerations are equal to infinity theoretically (graphs have been showed by thin arrows). The drive 1 should overcome theoretically infinitely big forces of inertia in these points. The adaptive drive 2 is capable to change independently the speed of motion under the acting of inertia forces of resistance. The initial inertial loading is forcing to increase the motion speed smoothly (not by jerk). The graph of accelerations for an adaptive drive 2 (displayed by a contour line) has only final values which call considerably smaller inertia forces of resistance. Therefore the adaptive drive should have considerably smaller power. For the manipulator the power of a drive is the major power characteristic defining a designer optimality because the sizes and module weight depend on power of its drive.

The drive with "rigid" constraint 1 provides motion with constant speed on all three phases of motion during a time T. Acceleration theoretically attains infinitely great value in the beginning and in the end of operation. The inertial force accepts values conforming to acceleration. Thus the engine of the drive 1 must overcome very big loading at start. Power of an engine must fit to starting loading.

The adaptive drive 2 is operating with brand new technology. At start the big inertia force allows to begin motion with a speed equal to null. Further the inertial force decreases, and speed of motion increases. The schedule of speed at start approaches to a cosine curve. The acceleration schedule at start approaches to a sinusoid. The inertial force accepts values conforming to acceleration. Thus the engine of the drive 2 must overcome extremely smaller loading at start. The maximum inertial force will be finite quantity and will occur approximately in the middle of start. Power of an engine should conform to this starting loading. Without account of minor factors acting on a draught resistance it is possible to assume, that the engine of the adaptive drive 2 can have power 10 times less in comparison with the engine of the usual drive 1 . Sizes and engine weight conform to its power. The adaptive drive will have small sizes and weight and high power efficiency because of possibility of adaptation to loading.

On the kinematic schedules (figure 1) it is visible a time of performance of each operation by the adaptive drive and by the usual drive equally. Displacements in the end of in stroke and in the end of backward stroke also are equal. The adaptive drive provides the variable transfer ratio inside of cycle at conservation of final displacements of forward stroke and of backward stroke. Therefore accuracy of final positioning of highprecision manipulator with the adaptive drive and with the usual drive will be equal.

Adaptation of the drive to a variable load leads to decrease of dynamic stresses (to unaccented work and elimination of vibrations).

Advantages of the drive 2 with the variable transfer ratio are indisputable. However practical implementation on the basis of existing designs in the form of the operated gear box is an insolvable problem for the robot.

However this problem is solved simply enough if to use an adaptive wheelwork with two degrees of freedom as the drive connecting gear [1, 2]. Such mechanism, first, is extremely simple on the device and, secondly, does not demand a control system. Change of the transfer ratio occurs in the adaptive mechanism independently depending on external loading.

Let's consider concrete circuit designs of adaptive drives of modules of the manipulator. 


\section{Design of the Adaptive Drive}

The adaptive module of translational motion (figure 2) contains following parts:

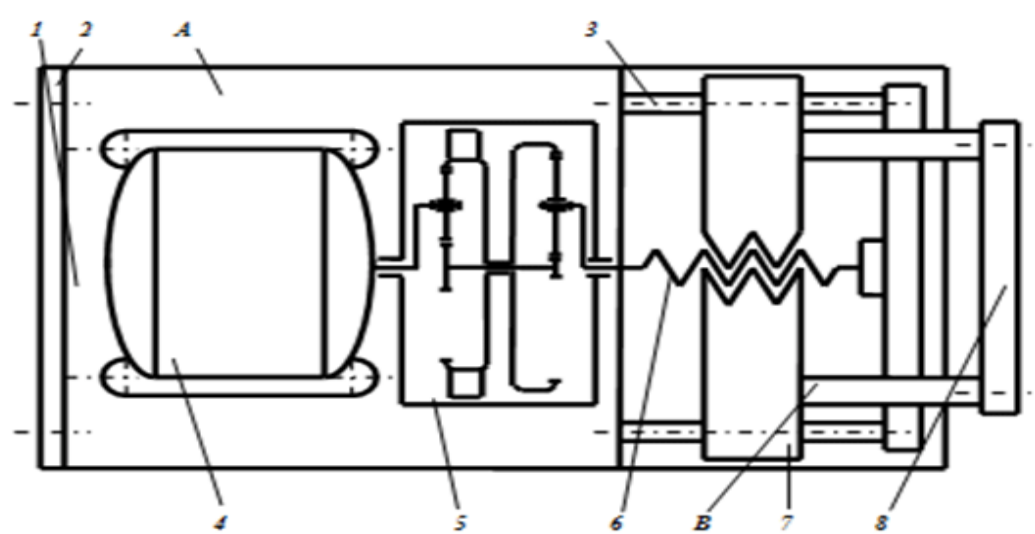

Figure 2: Adaptive module of translational motion of manipulator

$\boldsymbol{A}$ - module fixed part: 1. Plate. 2. Joint for junction with previous module 3. Directing for screw nut. 4. Electric motor. 5. Adaptive reducer. 6. Working screw.

$\boldsymbol{B}$ - module moving part: 7. Running screw nut of translational motion. 8. Joint on running screw nut for junction with the subsequent module.

The adaptive module of rotary motion (figure 3) contains following parts:

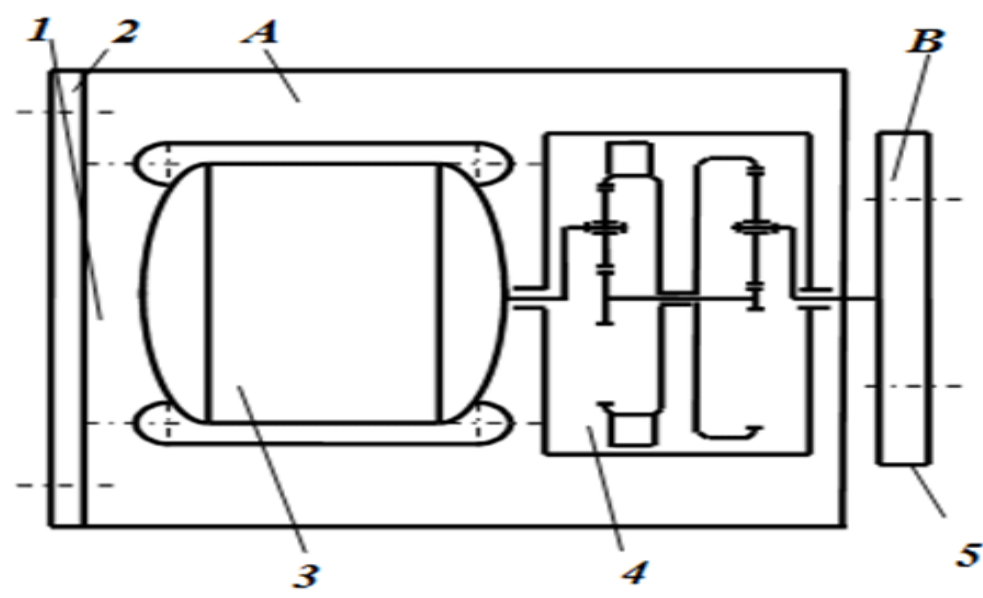

Figure 3: Adaptive module of rotary motion of manipulator

$\boldsymbol{A}$ - module fixed part: 1. Plate. 2. Joint for junction with previous module 3. Electric motor. 4. Adaptive reducer transferring rotary motion on an exit of module.

$\boldsymbol{B}$ - module moving part (exit of module). 5. Joint for junction with the subsequent module.

Adaptive mechanism (reducer) (figure 4) contains frame 0, input carrier H1, input satellite 2, block of central wheels 1-4, block of epicycle (ring) wheels 3-6 with flywheel on wheel 3, the output satellite 5 and output carrier H2. In operating time links are rotated round the central axis Sizes of toothed wheels 1, 2, 3, 4, 5, 6 are defined by conforming radiuses $r_{i} \quad i=1,2,3,4,5,6$ Radiuses of carriers $r_{H 1}=r_{1}+r_{2} r_{H 2}=r_{4}+r_{5}$. Number of degrees of freedom of the kinematic chain is equal 2 .

Adaptive mechanism (reducer) has variable transfer ratio which depends on loading on output shaft.

\section{Work of the Adaptive Drive}

In the drive of module of translational motion (figure 2) electric motor with constant power 4 transfers rotation to working screw 6 which moves a running screw nut 7 and module moving part B. Working screw 6 moves running screw nut 7 together with a module moving part B on

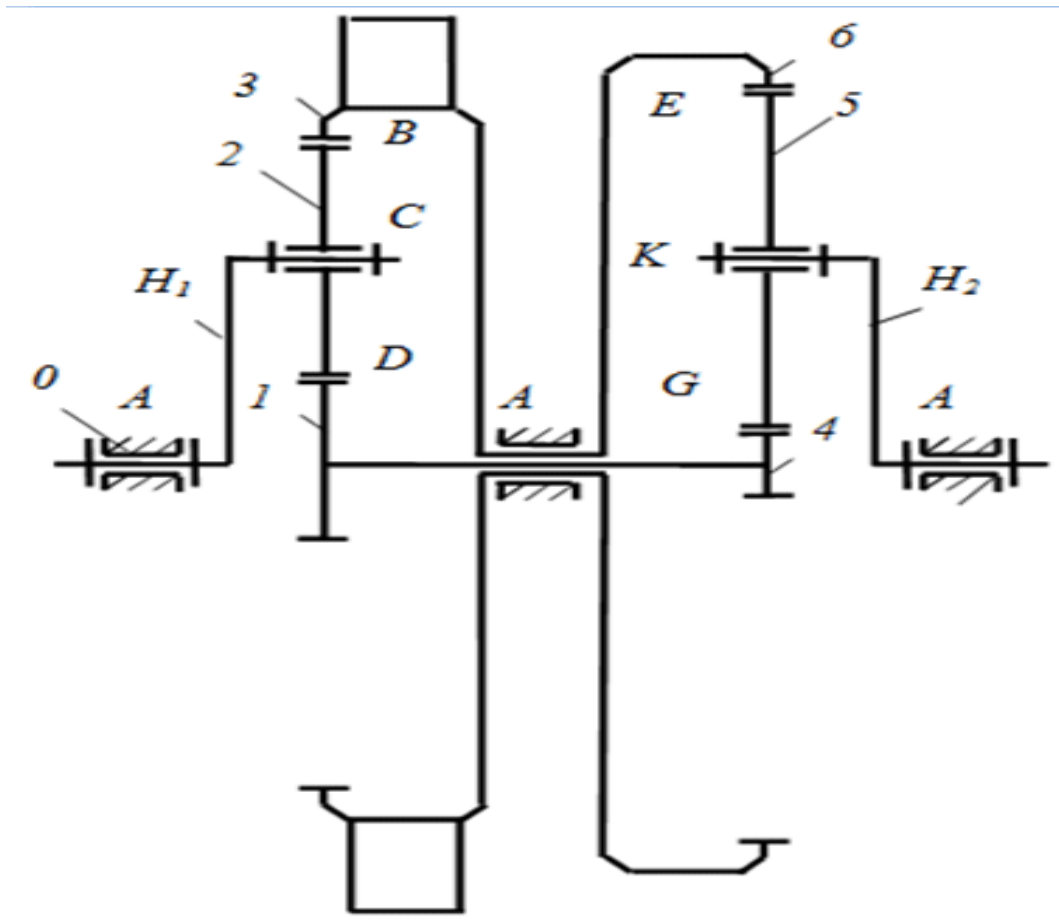

Figure 4: Adaptive mechanism of manipulator module drive

directions 3 . The running nut 7 together with attached to it the joint 8 carries out the output translational motion. Adaptive reducer 5 provides rotation of working screw 6 with a speed inversely proportional to moment of resistance which conforms to external loading of the module. The external force of resistance on the joint 8 independently changes speed of its translational motion.

Equations of kinematics of translational motion module define connection of linear motion of running screw nut 7 and output joint 8 with rotation of shaft of electric motor 4.

Speed of motion of running screw nut and moving part of module $V_{7}=n_{H 2} \cdot p$.

Here $n_{H 2}$ revolutions per minute of output carrier $\mathrm{H} 2$ of output shaft of adaptive reducer, P- step of nut screw line. Here $n_{H 2}=n_{H 1} / u\left(n_{H 1}\right.$ Her revolutions per minute of input carrier $H_{1}$ of electric motor shaft, $u$-variable transfer ratio of adaptive reducer). Then $V_{7}=n_{H 1} \cdot p / 60 \cdot u$.

Linear moving of running screw nut $S_{7}=n_{H 1} \cdot p \cdot t / 60 \cdot u$, where $t$ - time.

The sensing transducer of linear moving operates by module motion under the set program.

In the drive of rotary motion module (figure 3 ) electric motor 3 transfers rotation to the adaptive reducer 4 , moving part of module B and output Joint 5 . The adaptive reducer 4 provides rotation of output joint 5 with a speed inversely proportional to moment of resistance which conforms to external loading of the module. The external moment of resistance on the joint 5 independently changes speed of its rotary motion.

Equations of kinematics of the rotary motion module are equations of reducer 4 [1]. They define connection of angular motion of output joint 5 with rotation of electric motor shaft 4 .

The sensing transducer of angular moving operates by module motion under the set program.

\section{Kinematic and Force Analysis of Adaptive Mechanism of Manipulator Module}

In presented work unlike the usual mechanism the considered mechanism contains the mobile closed contour which connects input link with output link. This closed contour creates circulation of energy in a contour $[3,4]$. Energy circulation represents a connection equation of forces and speeds in the contour. This additional constraint reduces number degree 
of freedom to 1 and provides the installed regime of motion. Simultaneously the mobile closed contour of the mechanism creates the property of force adaptation.

Such is essence of the discovery which is the basis of the force adaptation. We will result here the new regularity.

At first we will consider the adaptive mechanism of manipulator module in the condition with two degrees of freedom.

The mechanism looks like the closed gear differential with two degrees of freedom (figure 4). Toothed wheels 1-2-3-6-5-4 are forming a closed four link contour. Input motive force $F_{1}$ is transferred from input link $H_{1}$ to point B. Output resistance force $R_{6}$ is transferred from output link $H_{2}$ to point $\mathrm{K}$. Application points $\mathrm{B}$ and $\mathrm{K}$ of contour external forces $F_{H 1}$ and $R_{H 2}$ have external displacements $\mathrm{S}_{\mathrm{B}}, \mathrm{S}_{\mathrm{K}}$. Application points $\mathrm{C}, \mathrm{E}, \mathrm{D}$, G of contour internal forces (reactions $R_{32^{\prime}} R_{65^{\prime}} R_{12^{\prime}} R_{45}$ ) have internal displacements $S_{C^{\prime}} S_{E^{\prime}} S_{D^{\prime}} S_{G^{\prime}}$ At known external displacements of points B and $\mathrm{K}$ the contour internal displacements of points SC, SE, SD, SG are defined as one-valued functions.

For each of contour links 2 and 5 the internal forces $R_{32^{\prime}} R_{65} R_{12^{\prime}} R_{45}$ can be expressed on statics conditions through active forces $\mathrm{FH}_{1}$ and $R \mathrm{H}_{2}$.

Let's make for links of 2 and 5 a conditions of equilibrium by principle of virtual works accepting the valid displacements for the possible.

For contour links 2 and 5 we will express reactions in the kinematic pairs D, C, G, E through external forces $\mathrm{FH}_{1}, \mathrm{RH}_{2}$ applied in points $\mathrm{B}, \mathrm{K}$

$$
\begin{aligned}
& R_{12}=R_{32}=0.5 F_{H 1} \\
& R_{45}=R_{65}=0.5 R_{H 2}
\end{aligned}
$$

Here

$$
F_{H 1}=M_{H 1} / r_{H 1}
$$

And so on

$R_{12}=M_{12} / r_{1}, R_{32}=M_{32} / r_{3}, R_{H 2}=M_{H 2} / r_{H 2}, R_{45}=M_{45} / r_{4}, R_{65}=M_{65} / r_{6}$,

$R_{H 2}=M_{H 2} / r_{H 2}, R_{45}=M_{45} / r_{4}, R_{65}=M_{65} / r_{6}$

$M_{H 1}, M_{H 2}$-Moments on input and output carriers,

$r_{H 1}, r_{H 2}$-Radiuses of input and output carriers,

$M_{12}, M_{32}$-Moments created on satellite 2 by reactions $R_{12}, R_{32}$ from toothed wheels 1 and 3,

$M_{45}, M_{65}$-Moments created on satellite 5 by reactions $R_{45}, R_{65}$ from toothed wheels 4 and 6 ,

$r_{i}(i=1,2 \ldots 6)$ - Radiuses of wheels.

After substitution of force values in the Eq. 1, Eq. 2 we will receive a formulas for determination of internal moments through the external moments

$$
\begin{aligned}
& M_{12}=0.5 M_{H 1} r_{1} / r_{H 1}, \\
& M_{32}=0.5 M_{H 1} r_{3} / r_{H 1}, \\
& M_{45}=0.5 M_{H 2} r_{4} / r_{H 2}, \\
& M_{65}=0.5 M_{H 2} r_{6} / r_{H 2},
\end{aligned}
$$

Equilibrium equations by a principle of virtual works for satellites 2 and 5 will have the following forms:

$$
\begin{aligned}
& R_{12} s_{D}+R_{32} s_{C}=F_{H 1} s_{B} \\
& R_{45} s_{G}+R_{65} s_{E}=R_{H 2} s_{K} .
\end{aligned}
$$

Here $s_{B}, s_{C}, s_{D}, s_{E}, s_{G}, s_{K}$ - displacements of points $B, C, D, E, G, K$.

The displacements of points expressed through instant angles of turn of links and radiuses will have the following forms:

$$
s_{D}=\phi_{1} r_{1}, s_{C}=\phi_{3} r_{3}, s_{B}=\phi_{H 1} r_{H 1}, s_{G}=\phi_{4} r_{4}, s_{E}=\phi_{6} r_{6}, s_{K}=\phi_{H 2} r_{H 2},
$$

$\phi_{1}, \phi_{3}, \phi_{H 1}, \phi_{4}, \phi_{6}, \phi_{H 2}$ - instant angles of turn of toothed wheels and carriers.

With account $\phi_{1}=\phi_{4}, \phi_{3}=\phi_{6}$ a time the equations (8) and (9) will receive following forms:

$$
\begin{aligned}
& M_{12} \omega_{1}+M_{32} \omega_{3}=M_{H 1} \omega_{H 1} \\
& M_{45} \omega_{1}+M_{65} \omega_{3}=M_{H 2} \omega_{H 2}
\end{aligned}
$$

To receive a condition of parameters interacting of the mechanism as a whole it is necessary to add the made expressions as satellites 2 and 5 are a part of the mechanism as a whole.

$$
M_{12} \omega_{1}+M_{32} \omega_{3}+M_{45} \omega_{1}+M_{65} \omega_{3}==M_{H 1} \omega_{H 1}+M_{H 2} \omega_{H 2}
$$

In the left side of Eq.12 the sum of powers (corresponding to the sum of works) of contour internal forces takes place.

In the considered mechanism all internal forces are determined through the known superposed forces and all internal displacements are determined through external displacements. Hence work (or power) of internal forces on possible internal displacements is determined. Constraints in kinematic pairs are ideal and fixed. Work of external forces cannot pass in work of internal forces. Hence work (power) of internal forces on possible internal displacements is equal to null

$$
M_{12} \omega_{1}+M_{32} \omega_{3}+M_{45} \omega_{1}+M_{65} \omega_{3}=0 .
$$

The right side of Eq.12 represents the sum of powers (corresponding to the sum of works) of contour external forces. At performance of Eq.33 we will receive from the Eq. 12 a condition of equilibrium for external forces according to principle of possible works

$$
M_{H 1} \omega_{H 1}+M_{H 2} \omega_{H 2}=0
$$

Equation (14) expresses the additional constraint imposed by a contour on motion of links.

Thus moving four link closed contour imposes a constraint on motion of links.

Additional constraint Eq.14 provides:

1) transformation of the kinematic chain with two degrees of freedom in the mechanism with one degree of freedom that is definability of motion under the action of forces;

2) effect of force adaptation to output loading at the assigned parameters of input power $M_{H 1}, \omega_{H 1}$ and the assigned output moment of resistance $M_{H 2}$.According to the Eq.14 with account signs of powers we will receive

$$
\omega_{H 2}=M_{H 1} \omega_{H 1} / M(15)
$$


That is at constant input power the output angular speed $\omega_{H_{2}}$ is inversely proportional to the variable output resistance moment $M_{H_{2}}$.

From the equation (13) it is possible to receive

$$
\left(M_{12}+M_{45}\right) \omega_{1}+\left(M_{32}+M_{65}\right) \omega_{3}=0 .
$$

With account signs of moments (the motive moments $M_{12}, M_{32}$ transferred from the input satellite 2 are positive, the resistance moments $M_{45}, M_{65}$ transferred from the output satellite 5 are negative) it follows

$$
\left(M_{12}-M_{45}\right) \omega_{1}+\left(M_{32}-M_{65}\right) \omega_{3}=0
$$

Eq.16 represents the equation of works (powers) on intermediate links 1-4 and 3-6. Eq. 16 means the presence of equilibrium on intermediate links 1-4 and 3-6 simultaneously. In the moving closed contour basic new situation takes place: equilibrium in statics is absent separately on each intermediate link but equilibrium of intermediate links simultaneously takes place on the movement all contour.

In the closed contour energy circulation takes place.

Eq.16 contains positive and negative members and characterizes equilibrium of powers on intermediate links of a contour. For the considered chain the follow constraint takes place

$$
\begin{gathered}
M_{45}>M_{12}, M_{32}>M_{65} \text {. Then from Eq.16 it is follows } \\
-\left(M_{45}-M_{12}\right) \omega_{1}+\left(M_{32}-M_{65}\right) \omega_{3}=0 . \text { (17) }
\end{gathered}
$$

From here

$$
\left(M_{45}-M_{12}\right) \omega_{1}=\left(M_{32}-M_{65}\right) \omega_{3} .
$$

Eq.18 reflects an analytical form of circulation of energy in a contour during its motion unknown earlier.

Angular speeds $\omega_{1}, \omega_{3}$ of intermediate links 1-4 and 3-6 are determined through known angular speeds of input and output carriers $\omega_{H 1}, \omega_{H 2}$ and transfer ratios at the stopped carriers.

Transfer ratios of links of mechanism we will determine through teeth numbers of wheels $z_{i} i=1,2, \ldots 6$. The interconnection of angular speeds of the mechanism is defined by formulas

$$
\begin{aligned}
& \frac{\omega_{1}-\omega_{H 1}}{\omega_{3}-\omega_{H 1}}=u_{13}^{(H 1)}, \\
& \frac{\omega_{1}-\omega_{H 2}}{\omega_{3}-\omega_{H 2}}=u_{46}^{(H 2)},
\end{aligned}
$$

where, $u_{46}^{(H 2)}=-z_{6} / z_{4}$. From (19)

$$
\omega_{1}=u_{13}^{(H 1)}\left(\omega_{3}-\omega_{H 1}\right)+\omega_{H 1} .
$$

From Eq.20

$$
\omega_{1}=u_{46}^{(H 2)}\left(\omega_{3}-\omega_{H 2}\right)+\omega_{H 2} .
$$

After of subtraction Eq.22 from Eq.21 it follows

$$
u_{13}^{(H 1)}\left(\omega_{3}-\omega_{H 1}\right)+\omega_{H 1}-u_{46}^{(H 2)}\left(\omega_{3}-\omega_{H 2}\right)-\omega_{H 2}=0 .
$$

From here

$$
\left(u_{13}^{(H 1)}-u_{46}^{(H 2)}\right) \omega_{3}-u_{13}^{(H 1)} \omega_{H 1}+u_{46}^{(H 2)} \omega_{H 2}=\omega_{H 2}-\omega_{H 1} .
$$

Eq.15, Eq.13 and Eq.21 define sequence of acts for definition angular speeds $\omega_{H_{2}}, \omega_{3}, \omega_{1}$ of mechanism links. It is necessary to note that at start-up the kinematic chain will move in a condition with one degree of freedom in the absence of mobility in a contour before performance of condition $M_{H_{2}}>M_{H 1}$. Thus all kinematic and force parameters are determined, and all mechanism has the kinematic and static definability.

The positioning problem should be solved by usual method - by using of feedback in a control system of module motion. The feedback sensor prolongs or shortens a time of operation performance. Hence, basic new problems of positioning of the adaptive drive are absent.

\section{Testing of the Adaptive Drive}

The adaptive drive has been tested at the stand (figure 5) [5].

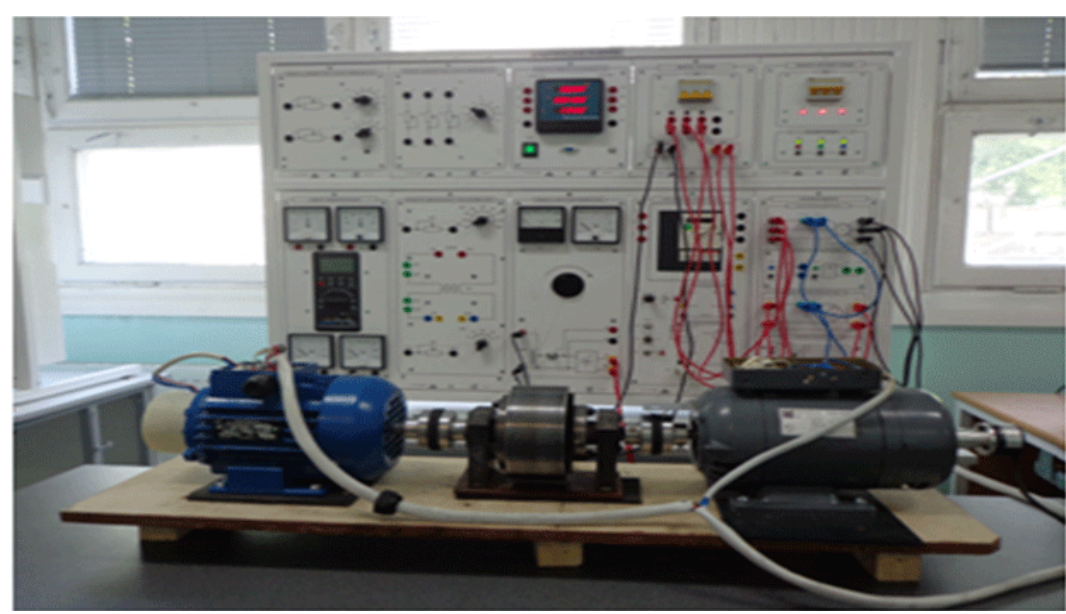

Figure 5: Stand for test adaptive drive

At the stand adaptive drive contains electric motor 1 and adaptive-mechanical transfer 2. Electric generator 3 simulates useful output loading. Theoretical regularities of adaptive drive are conforming to test results. Adaptive drive creates effect of force adaptation - at constant power of electric motor 1 angular speed of output shaft of reducer 2 is inverse proportional with variable loading which is creating by electric generator 3. Definiteness of reducer motion takes place both as in condition with two degrees of freedom (in operating regime) and in a condition with one degree of freedom (at start-up).

Work of the adaptive drive conforms to its tractive characteristic (figure 6) which has been gained at the drive experimental research on the test-bed.

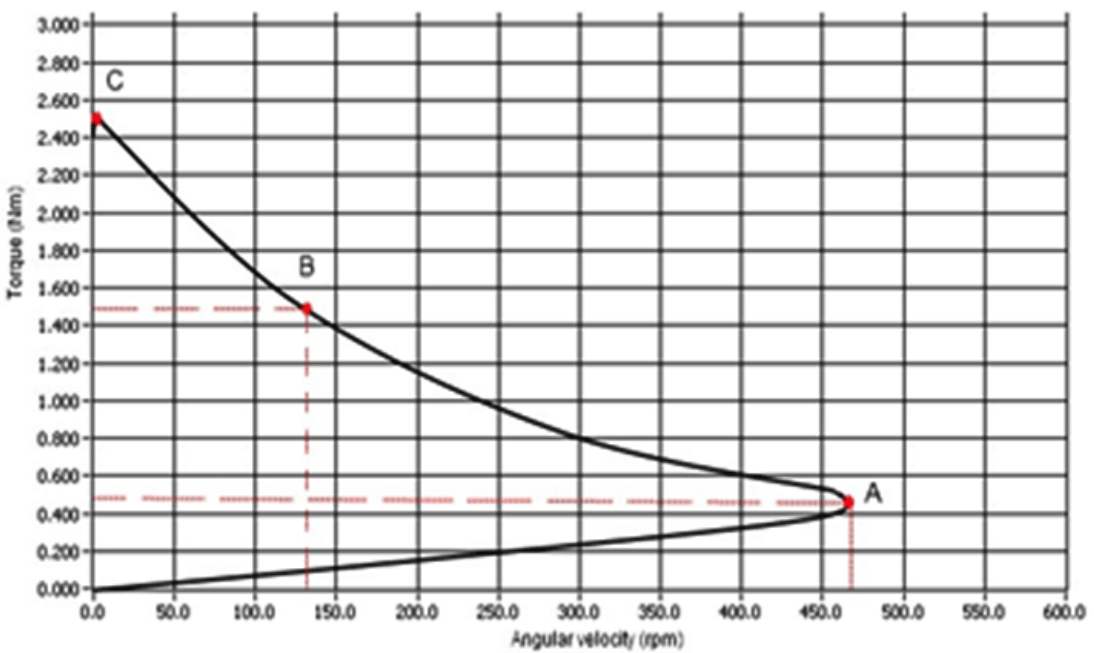

$O A$ - motion in condition with one degree of freedom in the absence of internal mobility into reducer; $\mathrm{ABC}$ - motion with two degrees of freedom (operating regime); $B$ - intermediate point; $C$ - operating regime end (maximum moment of resistance and stop).

Figure 6: Experimental tractive characteristic of adaptive reducer 
On figure 6 the experimental tractive characteristic of adaptive drive is presented. It has form of the schedule traction moment (torque) on output shaft of reducer depending on rotational speed of the shaft. Traction moment on reducer output shaft is equal to variable moment of resistance in operating regime. Adaptive reducer is set in motion by the electric motor of constant power at constant rotational speed of motor shaft. In the course of experiment no control means by power of the electric motor and its parameters were used.

In start-up regime driving moment of electric motor sweepingly changes from null to the rating value after switching of electric motor. Tractive characteristic of drive is presented by line which defines a startup regime in state with one degree of freedom. Adaptive reducer is moving as a single whole. The internal relative motion of wheels in the reducer is absent. Drive output shaft is revolution with rotation speed which is equal rated rotation speed of the electric motor. Tractive effort torque on output shaft of adaptive drive (in point of the characteristic) is equal to the moment on electric motor shaft $M_{H 2}=M_{H 1}=0.480 \mathrm{Nm}$. Rotational speed of the output shaft of reducer is equal to rotational speed of motor shaft $n_{H 2}=n_{H 1}=460 R P M$.

The operating regime of motion begins in point $A$ of curve $A B C$ when the output moment of resistance begins to exceed nominal tractive effort torque $M_{H_{2}}>M_{H 1}=0.480 \mathrm{Nm}$. In this case the reducer passes in state with two degrees of freedom. The power adaptation takes place. The output shaft rotational speed independently changes inversely a moment of resistance. The input moment of motor and its input rotational speed remain without change. They are equal to conforming rating values of parameters of the electric motor.

For example, in point $B$ traction moment on output shaft and conforming moment of resistance has the value $M_{H 2}>M_{H 1}=0.480 \mathrm{Nm}$. the rotational speed of output reducer shaft is equal $n_{H_{2}}=140 \mathrm{RPM}$

The maximum traction moment on reducer output shaft takes place in point $C$. The maximum traction moment is equal to maximum moment of resistance $M_{H 2}=2.500 \mathrm{Nm}$. The output shaft rotational speed the approach to point $C$ turn a minimum $n_{H 2}=8 R P M$. Then in point $C$ occurs a stop of the reducer output shaft $n_{H 2}=0$.Input shaft of reducer continues to rotation with rated rotation speed of electric motor $n_{H 1}=470 R P M$ The reducer passes in a state with one degree of freedom when the input shaft is rotation, and the output shaft is stop. So-called stop regime takes place.

Theoretically the reducer output power is equal to electric motor input power without a friction loss in operating regime of motion. At experiment conducting it was found out, that the increase in the traction moment leads to some decrease of an output power. It is connected with increase in relative speeds of rotation of wheels in the closed contour and increase in a friction loss.

\section{Calculation of Interconnecting Parameters of Adaptive Reducer}

Algorithm of adaptive reducer calculation conforms to earlier gained regularities [5]. Calculation is carried out in the set range of change of variable output torque $M_{H 2}$. form.

Algorithm of calculation of the adaptive reducer (figure 4) has next

Initial data: input angular speed $\omega_{H 1}$, input driving moment $M_{H 1}$ mand variable output torque $M_{H 2}$, numbers of teeth of wheels $z_{i}, \quad i=1,2,3,4,5,6$ module of engagement $m=1$.

It is necessary to determine the next parameters: angular speeds of output carrier and blocks of wheels 1-4 and 3-6 $\omega_{H 2}, \omega_{1}, \omega_{3}$ and moments of forces acting on wheels 1, 3, 4, $6 M_{12}, M_{32}, M_{45}, M_{65}$.
Solution.

1. Radiuses of toothed wheels $r_{i}=m z_{i} / 2$

2. Radiuses of carriers $r_{H 1}=\left(r_{1}+r_{3}\right) / 2, r_{H 2}=\left(r_{4}+r_{6}\right) / 2$

3. Transfer ratios in mechanism $u_{13}^{(H 1)}=-z_{3} / z_{1}, u_{46}^{(H 2)}=-z_{6} / z_{4}$

4. Angular speed of output carrier $\omega_{H 2}=M_{H 1} \omega_{H 1} / M_{H 2}$.

5. Angular speed of block of wheels 3-6 $\omega_{3}=\frac{\omega_{H 2}\left(1-u_{46}^{(H 2)}\right)-\omega_{H 1}\left(1-u_{13}^{(H 1)}\right)}{u_{13}^{(H 1)}-u_{46}^{(H 2)}}$

6. Angular speed of block of wheels 1-4 $\omega_{1}=u_{13}^{(H 1)}\left(\omega_{3}-\omega_{H 1}\right)+\omega_{H 1}$.

7. Moments on wheels 1, 3, 4, 6: $M_{12}=0.5 M_{H 1} r_{1} / r_{H 1}, M_{32}=0.5 M_{H 1} r_{3} / r_{H 1}$,

$$
M_{45}=0.5 M_{H 2} r_{4} / r_{H 2}, M_{65}=0.5 M_{H 2} r_{6} / r_{H 2}
$$

8. Check of balance of circulating energy. Here

$$
\begin{aligned}
& M_{65}>M_{32}, M_{12}>M_{45}, \\
& \left(M_{12}-M_{45}\right) \omega_{1}=\left(M_{65}-M_{32}\right) \omega_{3}
\end{aligned}
$$

If point 8 is carried out then performance of a following step of calculation algorithm at the next value of output torque take place. It is continued till using of end value of the rate.

Numerical check of algorithm of calculation of an adaptive wheelwork for one of values of output torque

It is given: $\omega_{H 1}=100 \mathrm{~s}^{-1}, M_{H 1}=15 \mathrm{Nm}, M_{H 2}=37.5 \mathrm{Nm}$,

$z_{1}=20, z_{2}=20, z_{3}=60, z_{4}=80, z_{5}=20, z_{6}=120, m=1 \mathrm{~mm}$

It is necessary to define the next parameters: $\omega_{H 2}, \omega_{1}, \omega_{3}, M_{12}, M_{32}, M_{45}, M_{65}$

Solution.

1. $r_{1}=m z_{1} / 2=1 \cdot 20 / 2=10, r_{2}=10, r_{3}=30, r_{4}=40, r_{5}=10, r_{6}=60$,

2. $r_{H 1}=\left(r_{1}+r_{3}\right) / 2=(10+30) / 2=20, r_{H 2}=\left(r_{4}+r_{6}\right) / 2=50$

3. $u_{13}^{(H 1)}=-z_{3} / z_{1}=-60 / 20=-3 u_{46}^{(H 2)}=-z_{6} / z_{4}=-120 / 80=-1.5$

4. $\omega_{H 2}=M_{H 1} \omega_{H 1} / M_{H 2}=100 \cdot 15 / 37.5=40 \mathrm{~s}^{-1}$.

5. $\omega_{3}=\frac{\omega_{H 2}\left(1-u_{46}^{(H 2)}\right)-\omega_{H 1}\left(1-u_{13}^{(H 1)}\right)}{u_{13}^{(H 1)}-u_{46}^{(H 2)}}=\frac{40(1+1.5)-100(1+3)}{-3+1.5}=200 \mathrm{~s}^{-1}$

6. $\omega_{1}=u_{13}^{(H 1)}\left(\omega_{3}-\omega_{H 1}\right)+\omega_{H 1}=(-3)(200-100)+100=-200 s^{-1}$.

7. $M_{12}=0.5 M_{H 1} r_{1} / r_{H 1}=0.5 \cdot 15 \cdot 10 / 20=3.75 \mathrm{Nm}$,

$M_{32}=0.5 M_{H 1} r_{3} / r_{H 1}=0.5 \cdot 15 \cdot 30 / 20=11.25 \mathrm{Nm}$,

$M_{45}=0.5 M_{H 2} r_{4} / r_{H 2}=0.5 \cdot 37.5 \cdot 40 / 50=15 \mathrm{Nm}$,

$M_{65}=0.5 M_{H 2} r_{6} / r_{H 2}=0.5 \cdot 37.5 \cdot 60 / 50=22.5 \mathrm{Nm}$,

$8 .(3.75-15) \cdot(-200)=(22.5-11.25) \cdot 200,11.25=11.25$

Further there is a repetition of calculation with new value of the output torque. 


\section{Adaptive Gripper}

Gripping and holding of objects are key tasks for robotic manipulators. The development of universal grippers able to pick up unfamiliar objects of widely varying shapes and surfaces is a very challenging task. The authors of the letter Petcovic D and Pavlovic ND [6] have presented the new principle of a new universal gripper with adaptive shape morphing surfaces. The adaptive surfaces have the controllability by a compliant system with embedded actuators and sensors. The main sensing system has to be made of a conductive silicone rubber or foam. These are carbon-blank filled silicone materials with good senses properties whose electrical resistance is changed by compression. The implemented controllable system is able to morph shapes of the gripper to accommodate different objects. But such system has low reliability, efficiency and high complexity. It is offered to use for observed situations adaptive gripper.

Adaptive gripper (figure 7) has adaptive drive in the form of lever mechanism with the closed contour.

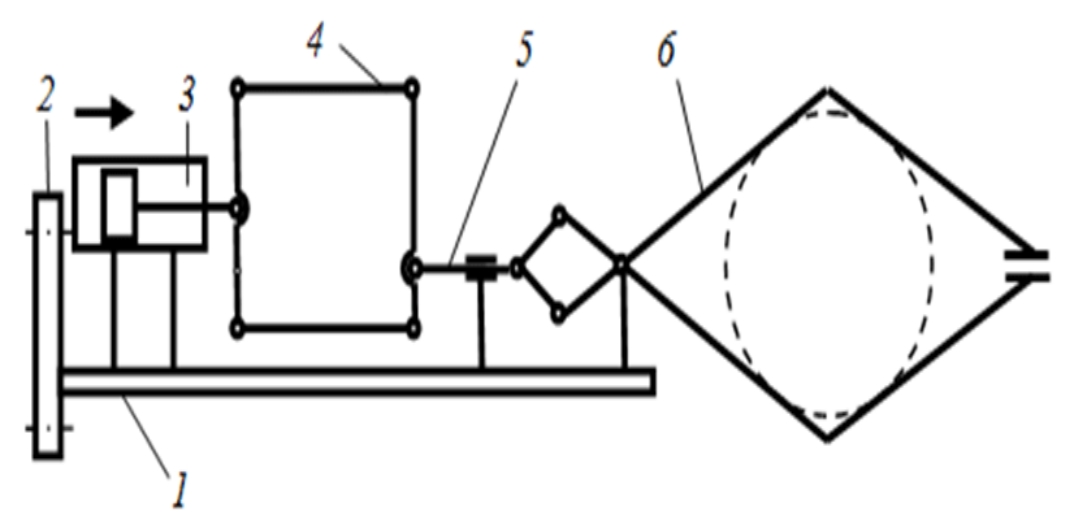

Figure 7: Adaptive gripper of the robot

Adaptive gripper contains basis 1 with joint 2, adaptive drive 3-4-5 and mechanism of gripper 6. Product in gripper is shown by a dot line Adaptive drive contains the electric solenoid 3 (or the hydro cylinder) and the adaptive lever mechanism with closed contour 4 .

The solenoid 3 moves input link of adaptive mechanism 4 to the right. The closed contour 4 transfers motion through output link 5 to the mechanism gripper 6 which provides a dripping of product. Force of dripping interferes with motion of link 5 and stops it at achievement of set value. Adaptive mechanism of drive passes in a condition with one degree of freedom. Then the position pickup switches out drive 3 and fixes it in final position. Product dripping by the adaptive mechanism occurs in state with two degrees of freedom without use of tactile sensing transducers. Gripper disclosing occurs at motion of input link of mechanism in the opposite direction.

\section{Adaptive Robot Transmissions in Extreme Working Conditions}

In many cases the robot is used for work in extreme conditions which create unpredictable and unexpected disturbances to motion in the form of repeatedly increased forces of resistance. Disturbances to motion can be called by environment (for example, space): the high temperature drop, vacuum, pressure, external mechanical affecting, etc. Disturbances call deformations of links, interacting conditions, break greasing conditions, lead to wedging. Disturbances are especially dangerous to the independent robots working without possibility of operative service. For work in extreme conditions the drive of the module with "rigid" kinematic constraint should be counted for the maximum possible draught resistance that leads to substantial growth of weight and sizes. The adaptive drive with adaptive kinematic constraint is capable to resist to the failures connected with an unforeseen overloading, without increase in power of an engine. For reliable overcoming of unforeseen disturbances motion is offered to use the vibrating adaptive drive which transfers vibration to moving links $[7,8]$. Vibration allows overcoming the increased forces of resistance at considerable decrease in power expenses.

The adaptive vibrating drive contains the adaptive planetary reducer (figure 4) in which the entrance satellite 2 has unbalanced weight, and blocks of wheels $1-4$ and $3-6$ have elastic constraints between wheels. During motion the unbalanced inertial force is transferred to the target carrier and calls torsion oscillations in the form of circular vibrations.

The adaptive vibrating drive has high power efficiency, small weight and sizes.

\section{Conclusions}

Regularity of interconnection of force and kinematic parameters of the robot in which each module has the adaptive electric drive are developed. The adaptive electric drive contains the electric motor and the adaptive connecting gear with two degrees of freedom. The adaptive mechanism provides motion of module moving part with a speed inverse to force loading at constant power of electric motor. The adaptive mechanism has the variable transfer ratio which depends on loading. Presence of definability of motion of the adaptive electric drive is confirmed by the drive experimental research. The experimental tractive characteristic containing a regime of start-up and operating regime is gained. The tractive characteristic conforms to the theoretical characteristic.

The adaptive drive is extremely simple in design. It changes the transfer ratio depending on loading independently only at the expense of mechanical properties and does not demand control of the transfer ratio. Power adaptation allows reducing sizes and weight of the drive at the expense of decrease of a required power of the electric motor. The equations of interconnection of parameters of motion of the adaptive mechanism are developed. The adaptive drive of the module of the robot provides a stop regime of motion at achievement of the maximum value of variable resistance. In this moment the module moving part appears as motionless and the electric motor continues motion with former speed. Stop regime allows to avoid an overloading and to overcome emergencies. In the robot with adaptive drives of modules stop regime can be overcome at the expense of motion of other modules leading to decrease of resistance.

In the article the demonstration of possibility of decrease of a required power of the engine is resulted. The adaptive module of translational motion and the rotary motion module are presented.

The use of the adaptive hydraulic drive allowing adapting for admitted force (for example, in gripper) is considered. The numerical instance of the drive calculation for confirming the force adaptation existence is resulted. The stand for testing of the adaptive drive is presented.

For reliable overcoming of unforeseen disturbances motion is offered to use the vibrating adaptive drive which transfers vibration to moving links. Vibration allows overcoming the increased forces of resistance at considerable decrease in power expenses.

All these materials should show the principal newness and efficiency of researches which are based on the science discovery.

The adaptive drive of the robot provides a high efficiency and has new abilities.

\section{References}

1. Brazhnikov AV, Babin VA, Belozerov IR. Fields of Possible and Optimum Application of "Combarco" Adaptive Variable-Speed Drives and Multiphase Asynchronous Inverter Systems. Modern high technologies. 2010;№ 2:20-23.

2. Fuchs RD, Hasuda Y, James IB. Full Toroidal IVT Variator Dynamics. SAE 2002 World Congress \& Exhibition. 2002.

3. Ivanov KS, Ualiev G, Tultaev B. Kinematic and Force Analysis of Robot with Adaptive Electric Drives. OPTIROB 2014. Springer. 2014:56-63. 
4. Ivanov KS. Action of Robot with Adaptive Electric Drives of Modules. Advances on Theory and Practice of Robots and Manipulators. 2014;22:563-569.

5. Ceccarelli M, Balbaev G, Ivanov K. An Experimental Test Validation of a New Planetary Transmission. International Journal of Mechanics and Control. 2014;15(2):1-7.

6. Petkovic D, Pavlovic ND. A New Principle of Adaptive Compliant Gripper. Mechanism and Machine Science 3. Mechanisms, Transmissions and Applications. 2012:3:143-150
7. Ivanov KS, Koilybayeva RK, Ualiev GU. Creation of Vibration Gear Continuously Variable Transmission (CVT). 11th International Conference on Vibration Problems (2013 ICOVP). 2013:91.

8. Ivanov KS, Koilibaeva RK, Ualiev GU, Tultaev BT. Creation of vibration gear continuously variable transmission (CVT) with one degree of freedom. 8th European Nonlinear Dynamics Conference (2014 ENOC). 2014:340. 